\title{
Cylindrical shapes of closed lipid bilayer structures correspond to an extreme area difference between the two monolayers of the bilayer
}

\author{
Aleš Iglič ${ }^{a}, *$, Veronika Kralj-Iglič ${ }^{b}$, Janja Majhenc ${ }^{\mathrm{b}}$ \\ ${ }^{a}$ Laboratory of Applied Physics, Faculty of Electrical Engineering, University of Ljubljana, Tražaška 25, SI-1000 Ljubljana, Slovenia \\ ${ }^{\mathrm{b}}$ Institute of Biophysics, Medical Faculty, University of Ljubljana, Lipičeva 2, SI-1000 Ljubljana, Slovenia
}

Accepted 22 June 1999

\begin{abstract}
The shapes of extreme area difference between the outer and the inner layer $(\Delta A)$ of the closed lipid bilayer structures at fixed membrane area $(A)$ and fixed volume $(V)$ are determined by stating and analytically solving a variational problem for axisymmetric shapes. It is shown that the spheres with at most two different radii and the cylinder are the solutions of this variational problem. The cylinder ended by a hemisphere on each end is the shape combined from these solutions and is therefore, itself the shape of the extreme $\Delta A$ at fixed $V$ and $A$. The related cylindrical shapes of stearoyl-oleoyl-phosphocholine vesicles are shown. (C) 1999 Elsevier Science Ltd. All rights reserved.
\end{abstract}

\section{Introduction}

The occurrence of red blood cell shape (RBC) changes and the vesiculation of RBC membrane have been observed in some human blood disorders (Palek, 1987; Wagner, 1986). Therefore better understanding of physical mechanisms determining RBC shape and RBC membrane vesiculation may help to devolop a new medical treatment to reduce some complications of blood disorders (Wagner, 1986). The human RBC has no internal structure, therefore its shape at given cell volume is determined solely by the physical properties of the cell membrane (Evans, 1974; Deuling and Helfrich, 1976). The red blood cell membrane is essentially composed of two parts, the bilayer and the continuous network of proteins - the membrane skeleton (Evans and Skalak, 1980). Although the RBC membrane is structurally and mechanically more complex than that of the closed bilayer lipid vesicles (Evans and Skalak, 1980; Sackmann, 1994; Iglič, 1997), the study of the shape changes of lipid vesicles can be directed to many aspects of the RBC behaviour (Svetina and Žekš, 1996).

The transformations beetwen the shapes of lipid vesicle or cell with no internal structure can be qualitatively well

\footnotetext{
* Corresponding author. Tel.: + 386-61-1768-411; fax: + 386-611264-630.

E-mail address: ales.iglic@fe.uni-lj.si (A. Iglič)
}

explained within the bilayer couple model (Sheetz and Singer, 1974; Evans, 1974). In the view of this model the difference between the outer and the inner membrane lipid layer area of the membrane $(\Delta A)$ is taken to be an important parameter which influences the cell shape (Svetina and Žekš, 1996; Seifert, 1997; Iglič et al., 1998b).

Within the bilayer couple model the class of cell or vesicle shapes of a fixed area $A$ contains all the stationary shapes of the same symmetry that can be continuously transformed one into other by continuously varying the two model parameters, i.e. the cell volume $V$ and the difference between the areas of the two membrane lipid layers $\Delta A$. The shapes of the extreme $V$ at given $A$ and $\Delta A$ (Svetina and Žekš, 1996) or the shapes of the extreme $\Delta A$ at given $V$ and $A$ (Iglič et al., 1998a) were defined as the shapes corresponding to the boundaries of certain classes of the phase diagram of possible shapes. We call these shapes the limit shapes (Seifert et al., 1991; Svetina and Žekš, 1996). It was shown analytically by means of solving a variational problem that the limit shapes can be composed of spheres or sections of spheres, where at most two different radii are possible (Svetina and Žekš, 1996; Iglič et al., 1998a). It was recently indicated that in addition to these, the limit shapes can be composed also from cylindrical and toroidal parts (Iglic et al., 1998a; Hägerstrand et al., 1998).

In this paper we theoretically describe the limit shape of closed bilayer structures composed of the cylinder ended with two hemispheres. Numerical calculations 
indicate the existence of such shapes at the boundary of the dumbbell class (Seifert et al., 1991; Svetina and Žekš, 1991). However, no analytical proof for the existence of the cylindical limit shape has yet been offered. In this work it is shown analytically as well as calculated numerically that the cylinders ended with hemispheres are the limit shapes of prolate class of shapes. Moreover, experimental evidence of the existence of such limit shapes in the case of stearoyl-oleoyl-phosphocholine (SOPC) vesicles is given.

\section{Materials and Methods}

\subsection{Experiment}

SOPC was purchased from Avanti Polar Lipids (Al, USA) and used without further purification. We dissolved the lipid powder in a mixture of chloroform and methanol $(1: 1 \mathrm{v} / \mathrm{v})$ to $1 \mathrm{mg} / \mathrm{ml}$ and stored it at $258 \mathrm{~K}$. Giant vesicles were prepared by modified method of Reeves and Dowben (Reeves and Dowben, 1969). Approximately $50 \mu \mathrm{l}$ of lipid solution was spread on the teflon disk and left for at least $2 \mathrm{~h}$ under low vacuum for the solvent to evaporate. The teflon disk with lipid film was placed at the bottom of $50 \mathrm{ml}$ beaker and exposed to a gentle stream of water saturated nitrogen for $30 \mathrm{~min}$. Then, $100 \mathrm{mM}$ glucose solution was added and left to stand for some days at room temperature. The suspension with SOPC vesicles, kept in a glass chamber at room temperature, was observed by a phase contrast microscope (Obj.Ph 3, NA 1.3, $100 \times$, Oil).

\subsection{Possible limit shapes of vesicles corresponding to extreme values of $\Delta A$}

In order to obtain the limit vesicle shape of an extreme $\Delta A$ (Eq. (9)) at a given $A$ and $V$, a variational problem is stated by constructing a functional (Iglič et al., 1998a)

$Q=\Delta A-\lambda_{\mathrm{A}}\left(A-\int \mathrm{d} A\right)-\lambda_{\mathrm{V}}\left(V-\int \mathrm{d} V\right)$,

where $\lambda_{\mathrm{A}}$ and $\lambda_{\mathrm{V}}$ are the Lagrange multipliers which can be determined from the constraints for the membrane area and the enclosed vesicle volume. The analysis is restricted to axisymmetric vesicle shapes where the symmetry axis of the vesicle coincides with the $x$-axis, so that the shape is given by the rotation of the function $y(x)$ around the $x$-axis. The variation $\delta Q=0$ is performed by solving the corresponding Euler-Poisson equation (Iglič et al., 1998a)

$$
\begin{aligned}
& 2 y^{\prime \prime}\left(1+y^{\prime 2}\right)^{-2}+\lambda_{\mathrm{A}}\left(\left(1+y^{\prime 2}\right)^{-1 / 2}-y y^{\prime \prime}\left(1+y^{\prime 2}\right)^{-3 / 2}\right) \\
& \quad-\lambda_{\mathrm{V}} y=0
\end{aligned}
$$

where $\lambda_{\mathrm{A}}$ and $\lambda_{\mathrm{V}}$ are renormalized. It has been shown (Iglic et al., 1998a) that the above Eq. (2) can be solved by the ansatz for the sphere with at most two different radii,

$R_{1,2}=2 /\left(\lambda_{\mathrm{A}} \pm\left(\lambda_{\mathrm{A}}^{2}-2 \lambda_{\mathrm{V}}\right)^{1 / 2}\right)$,

as well by the the ansatz $y=\lambda_{\mathrm{A}} / \lambda_{\mathrm{V}}$ representing the cylinder of the radius

$r=\lambda_{\mathrm{A}} / \lambda_{\mathrm{v}}$.

Different combination of the above solutions are possible, provided that the combined shape fulfills the constraints and possible additional conditions. One of the possible combined shapes of the extreme $\Delta A$ at fixed $V$ and $A$ is the cylinder ended by a hemisphere on each end of the cylinder. For such combined shape the Lagrange multipliers are interdependent, $\lambda_{\mathrm{A}}^{2}=2 \lambda_{\mathrm{v}}$. The shape is characterized by two parameters: the radius of the cylinder which is equal to the radius of the hemisphere $(r)$ and the length of the cylinder $(l)$ which can be determined from the contraints for the vesicle volume and area. As the number of the parameters in this case equals the number of the constraints, the cylinder ended with hemispheres fulfills the requirement for the limit shape corresponding to the extreme $\Delta A$ (Elsgolc, 1961).

To calculate the parameters of the cylinder ended with hemispheres, dimensionless quantities are introduced. For the unit length, the radius of a sphere $R_{\mathrm{S}}$ with the membrane area $A$ is chosen. In accordance with the definition of the radius $R_{\mathrm{S}}$, the relative area $a=A / 4 \pi R_{\mathrm{S}}^{2}$ is equal to one, the relative volume is $v=3 V / 4 \pi R_{\mathrm{S}}^{3}$ and the relative area difference is $\Delta a=\Delta A / 8 \pi h R_{\mathrm{S}}$. At a given relative volume $v$ the radius $r$ and the length of the cylinder $l$ can be determined from the constraints for the relative area and the relative volume of the vesicle:

$1=r^{2}+r l / 2$

$v=r^{3}+3 r^{2} l / 4$,

where $r$ and $l$ are measured in the units of $R_{\mathrm{S}}$.

In experimental procedures the relative area difference $\Delta a$ and the relative volume $v$ of the artificial lipid vesicles can be varied continuously by changing the temperature or osmotic conditions (Helfrich, 1973; Berndl et al., 1990; Käs and Sackmann, 1991; Seifert et al., 1991). Also, the relative area difference $\Delta a$ can be modified by redistribution of lipids from one monolayer to another by applying a transmembrane $\mathrm{pH}$ gradient (Farge and Devaux, 1992).

\section{Results}

Fig. 1 shows the values of $\Delta a$ of the cylinder ended with hemispheres in dependence of its relative volume $v$. It can be seen in Fig. 1 that the length of the cylindrical part of the vesicle $l$ increases while the radius $r$ decreases with 


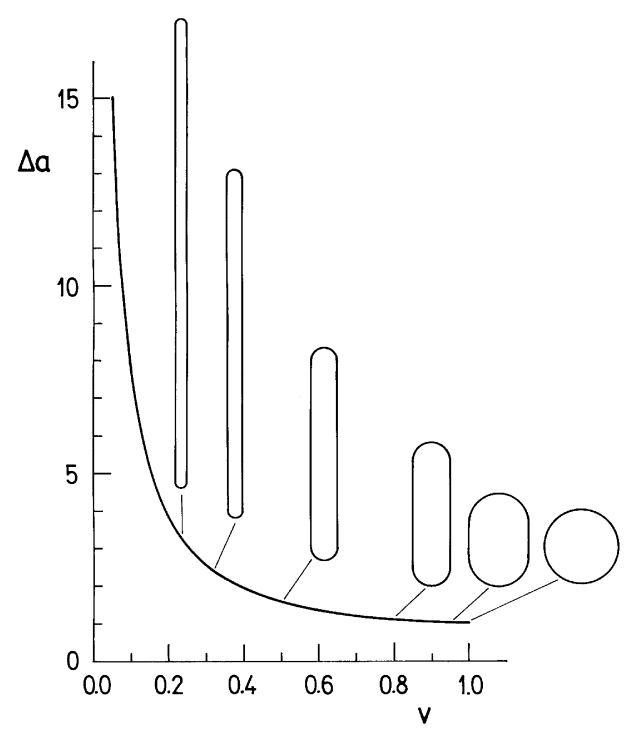

Fig. 1. Relative area difference $\Delta a$ of the cylindrical vesicle ended with hemispheres in dependence of the relative volume $(v)$. Figure also shows some characteristic vesicle shapes.

decreasing $v$. It can be also seen in Fig. 1 that the relative area difference $\Delta a$ increases with decreasing relative vesicle volume $v$. Consequently, the enclosed relative volume of the cylindrical vesicle ended with hemispheres should be as small as possible in order to render the value of $\Delta a$ the largest.

The above theory gives the shapes at the boundary of the phase diagram of the possible shapes. Due to confirmation it is of convenience to find a sequence of shapes within a given class of vesicle shapes that leads to the boundary of the phase diagram upon a continuous change of the relative area difference $\Delta a$. Fig. 2 shows that the cylindrical vesicle shape ended with hemispheres represents the lower limit of a sequence of the prolate vesicle shapes with equatorial mirror symmetry. The shapes B-E in Fig. 2 are obtained numerically by solving the variational problem of varying the local bending energy for the axisymmetric vesicle shape at fixed $A, V$ and $\Delta A$ (Deuling and Helfrich, 1976; Svetina and Žekšs, 1996; Seifert, 1997). The corresponding differential equation for the axisymmetric vesicle shape is given in the appendix. The shape $\mathrm{F}$ was calculated numerically from Eqs. (5) and (6) using the tangential method. The limit shape A was calculated analytically from the constraints for the relative area and the relative volume of the limit vesicle shape composed of three spherical vesicles with the same radius connected by the ideal necks.

The theoretically predicted cylindrical (prolate) shapes are compared with the observed ones. Fig. 3 shows the phase contrast micrograph of a SOPC cylindrical vesicle. The vesicle shape remained stable for at least half an hour. In Fig. 4 shape transformation from initially undulated shape (Fig. 4A) to a more cylindrical shape

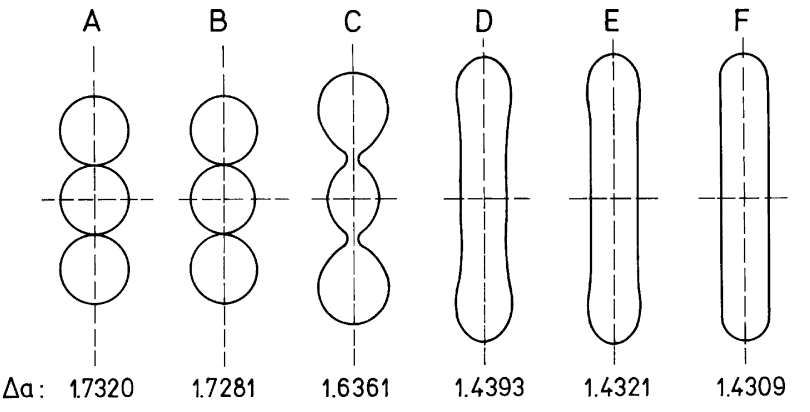

Fig. 2. The sequence of the prolate vesicle shapes with equatorial mirror symmetry for $v=3^{-1 / 2}$. The shape A represents the limit shape composed of spheres of equal radii while the the shape $\mathrm{F}$ is the limit shape composed of the cylinder ended with hemispheres on both ends.

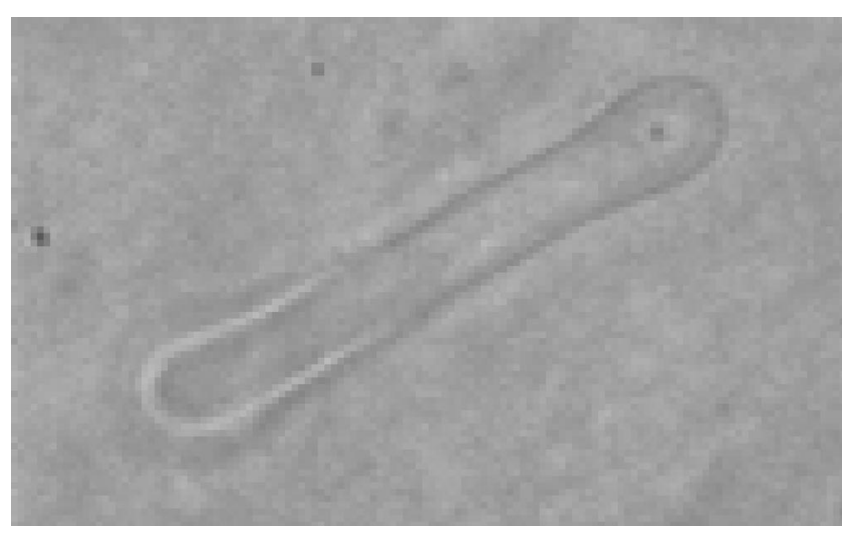

Fig. 3. Phase contrast micrograph of the SOPC cylindrical vesicle.
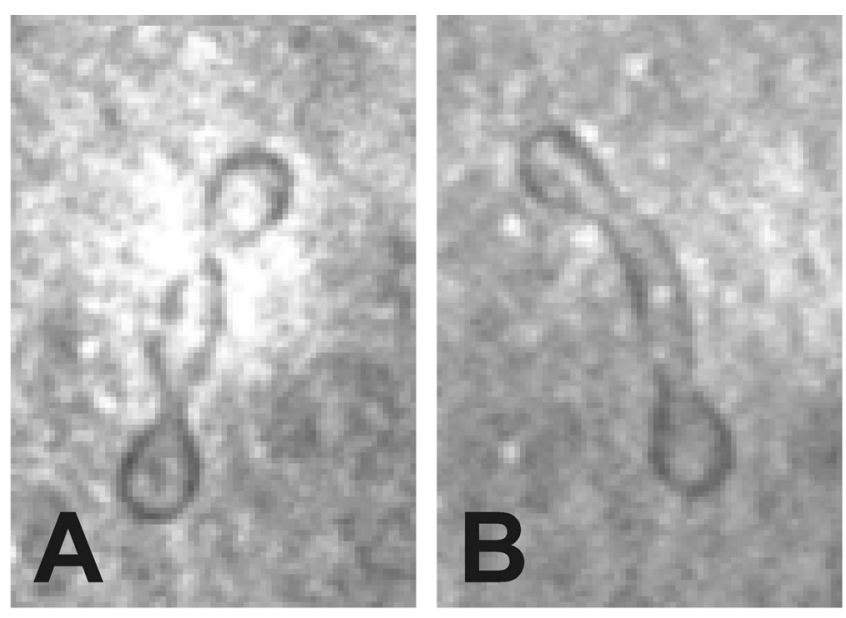

Fig. 4. Phase contrast micrographs of the SOPC prolate vesicle at two different times. The undulated shape of the vesicle (A) has larger $\Delta A$ than the cylindrical shape (B).

(Fig. 4B) took place within some minutes. We believe that the shape change occured due to the decrease of $\Delta A$ caused by the drag of the lipids from the outer membrane layer by the glass of the chamber. After the vesicle had reached the cylindrical shape it remained unchanged for half an hour and was no longer observed. It can be seen 
that good qualitative agreement between the observed shape transformations (Fig. 4) and the calculated shape transformations of the prolate undulated vesicle (Fig. 2) induced by decreasing of $\Delta A$ is obtained.

\section{Conclusions and Discussion}

The described limit cylindrical vesicle shape composed of a cylindrical part and two hemispheres renders a discontinuity of the principal curvature along the meridians at the junction between the cylindrical part and the spherical part (Fig. 2F). Therefore, in reality, the observed cylindrical vesicle shapes (Fig. 3) never reach the exact limit cylindrical shape ended with hemispheres. However, they are very close to the calculated shape E in Fig. 2 where the discontinuities at the cylindrical-spherical junction are smoothened out, i.e. the radius of the cylinder smoothly ends by two caps of a slightly enlarged extension perpendicular to the cylinder axis.

The prolate shapes of the vesicle at given $A, \Delta A$ and $V$ (Fig. 2) was determined by minimization of the local bending energy. Including the nonlocal bending energy (Evans and Skalak, 1980) in the minimization procedure would not change the calculated shape at given $\Delta A$, only the total energy of the vesicle membrane would be different (Svetina and Žekš, 1996; Iglič et al., 1998b). Including some other contributions to the membrane free energy such as the energy due to inhomogeneous distribution of the membrane components (Lipowsky, 1993; Kralj-Iglič et al., 1996) would influence also the calculated equilibrium vesicle shape at given $\Delta A$. However, the limit shape corresponding to the extreme $\Delta A$ would remain the same.

The spherical and the cylindrical limit shapes and their possible combinations are not the only possible limit shapes of the extreme $\Delta A$. We have recently shown that the torus and the torocyte shapes (Hägerstrand et al., 1998) are also the shapes of the extreme $\Delta A$.

In conclusion, we have shown analytically for the first time that the vesicle shape composed of the cylinder ended with two hemispheres is the limit vesicle shape corresponding to the extreme value of $\Delta A$. The related structures in the case of the SOPC vesicles were observed (Figs. 3 and 4). On the basis of the presented results it can be suggested that while describing the boundaries of the phase diagram of the vesicle shapes the cylindrical limit shapes and their combinations with spherical shapes should also be considered in addition to the previously described spherical and toroidal limit shapes.

\section{Acknowledgements}

The authors would like to thank Dr. Volkmar Heinrich, University of British Columbia, Vancouver, for software used in numerically solving the system of Lagrange-Euler equations.

\section{Appendix A}

The vesicle shape with minimal local bending energy (Deuling and Helfrich, 1976)

$W_{\mathrm{b}}=\frac{1}{2} k_{\mathrm{c}} \int\left(C_{1}+C_{2}\right)^{2} \mathrm{~d} A$.

at given $A, V$ and $\Delta A$ is obtained by minimizing the functional (Deuling and Helfrich, 1976; Svetina and Žekšs, 1996)

$F=W_{\mathrm{b}}-\lambda_{\mathrm{A}} A-\Lambda_{\mathrm{V}} V-\Lambda_{\Delta} \Delta A$,

where the Lagrange multipliers $\Lambda_{\mathrm{A}}, \Lambda_{\mathrm{V}}$ and $\Lambda_{\Delta}$ can be determined from the constraints for the area, volume and area difference. Here $k_{\mathrm{c}}$ is the local bending modulus of the membrane, $C_{1}$ and $C_{2}$ are the principal membrane curvatures. Integration in Eq. (7) is performed over the bilayer neutral surface area of the segment. Since the distance between the neutral surfaces of the bilayer leaflets $(h)$ is much smaller than the dimensions of the vesicle, the area difference $\Delta A$ can be written as

$\Delta A=h \int\left(C_{1}+C_{2}\right) \mathrm{d} A$.

where $C_{1}$ and $C_{2}$ are defined so that they are positive for a sphere.

In the following analysis dimensionless quantities are introduced. To describe the equilibrium shape, we introduce independent coordinates $\rho(s)$ and $z(s)$ (Jülicher and Seifert, 1994) where $\rho$ is the distance between the symmetry axis and a certain point on the contour, $z$ is the position of this point along the symmetry axis and $s$ is the arclength along the contour. These coordinates are normalized with respect to $R_{\mathrm{S}}$. The angle $\psi(s)$ made by surface normal and $z$ axis is defined by the equation tan $\psi=\mathrm{d} z / \mathrm{d} \rho$ (Deuling and Helfrich, 1976).

The bending energy $W_{\mathrm{b}}$ and the functional $L$ are normalized relative to the bending energy of the sphere $8 \pi k_{\mathrm{c}}$ :

$f=F / 8 \pi k_{\mathrm{c}}=w_{\mathrm{b}}-\lambda_{\mathrm{A}} a-\lambda_{\mathrm{v}} v-\lambda_{\Delta} \Delta a$,

where $w_{\mathrm{b}}=W_{\mathrm{b}} / 8 \pi k_{\mathrm{c}}$ and the new Lagrange multipliers are $\lambda_{\mathrm{A}}=\Lambda_{\mathrm{A}} R_{\mathrm{s}}^{2} / 2 k_{\mathrm{c}}, \lambda_{\mathrm{v}}=\Lambda_{\mathrm{v}} R_{\mathrm{s}}^{3} / 6 k_{\mathrm{c}}$ and $\lambda_{\Delta}=\Lambda_{\Delta} h R_{\mathrm{s}} / k_{\mathrm{c}}$. The restriction for the geometrical relations between the angle $\psi$ and the coordinate $\rho$ is taken into account by introducing an additional Lagrange multiplier $\gamma(s)$ (Heinrich, 1991; Jülicher and Seifert, 1994). Using the 
definition $f=\int L \mathrm{~d} s$, where

$$
\begin{aligned}
L= & \left(\rho(\mathrm{d} \psi / \mathrm{d} s)^{2}+\sin ^{2} \psi / \rho\right)+\lambda_{\mathrm{A}} \rho / 2+3 \lambda_{\mathrm{V}} \rho^{2} \sin \psi / 4 \\
& +\lambda_{\Delta}(\rho \mathrm{d} \psi / \mathrm{d} s+\sin \psi)+\gamma(\mathrm{d} \rho / \mathrm{d} s-\cos \psi),
\end{aligned}
$$

the Lagrange-Euler equations for the described variational problem can be then written as

$$
\begin{aligned}
\mathrm{d} \omega / \mathrm{d} s= & \sin \psi \cos \psi / \rho+3 \lambda_{\mathrm{V}} \rho^{2} \cos \psi+4 \gamma \sin \psi, \\
\mathrm{d} \gamma / \mathrm{d} s= & \left(\omega^{2}-\sin ^{2} \psi\right) / 8 \rho^{2}+\lambda_{\mathrm{A}} / 2+3 \lambda_{\mathrm{V}} \rho \sin \psi / 2 \\
& +\lambda_{\Delta} \omega / 4 \rho,
\end{aligned}
$$

In Eqs. (12)-(14) the function $\omega$ is defined as

$\omega=\rho \mathrm{d} \psi / \mathrm{d} s$

Eqs. (12)-(16) are solved numerically as described in detail elsewhere (Heinrich, 1991).

\section{References}

Berndl, K., Käs, J., Lipowsky, R., Sackmann, E., Seifert, U., 1990. Shape transformations of giant vesicles: extreme sensitivity to bilayer asymmetry. Europhysics Letters 13, 659-664.

Deuling, H.J., Helfrich, W., 1976. The curvature elasticity of fluid membranes. Journal de Physique II (France) 37, 1335-1345.

Elsgolc, L.E., 1961. Calculus of Variations. Pergamon Press, Oxford, pp. $1-82$.

Evans, E.A., 1974. Bending resistance and chemically induced moments in membrane bilayers. Biophysical Journal 14, 923-931.

Evans, E., Skalak, R., 1980. Mechanics and Thermodynamics of Biomembranes. CRC Press, Boca Raton, FL, pp. 1-254.

Farge, E., Devaux, P.F., 1992. Shape changes of giant liposomes induced by asymmetric transmembrane distribution of phospholipids. Biophysical Journal 61, 347-357.

Hägerstrand, H., Kralj-Iglič, V., Bobrowska-Hägerstrand, M., Bialkowska, K., Isomaa, B., Iglič, A., 1998. Torocytes - a new class of vesicle shapes. Cellular and Molecular Biology Letters 3, 145-150.

Heinrich, V., 1991. Theoretische Bestimmung von kugelnahen Vesikelformen mit Hilfe von Kugelfunktionen. Ph.D. Thesis, Humboldt University, Berlin.
Helfrich, W., 1973. Elastic properties of lipid bilayers: theory and posssible experiments. Zeitschrift für Naturforschung 29C, 693-703.

Iglič, A., 1997. A possible mechanism determining the stability of spiculated red blood cells. Journal of Biomechanics 30, 35-40.

Iglič, A., Hägerstrand, H., Kralj-Iglič, V., Bobrowska-Hägerstrand, M., 1998a. A possible physical mechanism of red blood cell vesiculation obtained by incubation at high $\mathrm{pH}$. Journal of Biomechanics 31 , $151-156$

Iglič, A., Kralj-Iglič, V., Hägerstrand, H., 1998b. Stability of spiculated red blood cells induced by intercalation of amphiphiles in cell membrane. Medical and Biological Engineering \& Computing 36, 251-255.

Jülicher, F., Seifert, U., 1994. Shape equations for axisymmetric vesicles: a clarification. Physical Review E 49, 4728-4731.

Käs, J., Sackmann, E., 1991. Shape transitions and shape stability of giant phospholipid vesicles in pure water induced by area-to-volume changes. Biophysical Journal 60, 825-844.

Kralj-Iglič, V., Svetina, S., Žekš, B., 1996. Shapes of bilayer vesicles with membrane embedded molecules. European Biophysics Journal 24, 311-321.

Lipowsky, R., 1993. Domain-induced budding of fluid membranes. Biophysical Journal 64, 1133-1138.

Palek, J., 1987. Hereditary elliptocytosis, spherocytosis and related disorders: consequences of a deficiency or mutation of membrane skeletal proteins. Blood Reviews 1, 147-168.

Reeves, J.P., Dowben, R.M., 1969. Formation and properties of thinwalled phospholipid vesicles. Journal of Cellular Physiology 73, 49-60.

Sackmann, E., 1994. Membrane bending energy concept of vesicle and cell shapes and shape transitions. FEBS Letters 346, 3-16.

Seifert, U., Berndl, K., Lipowsky, R., 1991. Shape transformations of vesicles: Phase diagram for spontaneous-curvature and bilayercoupling models. Physical Review A 44, 1181-1202.

Seifert, U., 1997. Configurations of fluid membranes and vesicles. Advances in Physics 46, 13-137.

Sheetz, M.P., Singer, S.J., 1974. Biological membranes as bilayer couples. A molecular mechanism of drug-erythrocyte interactions. Proceedings of the National Academy of Sciences (USA) 71, 4457-4461.

Svetina, S., Žekš, B., 1996. Elastic properties of closed bilayer membranes and the shapes of giant phospholipid vesicles. In: Lasic, D.D., Barenholz, Y. (Eds.), Handbook of Nonmedical Applications of Liposomes. CRC Press, Boca Raton, pp. 13-42.

Svetina, S., Žekš, B., 1991. Mechanical behaviour of closed lamellar membranes as a possible common mechanism for the establishment of developmental shapes. International Journal of Developmental Biology 35, 359-365.

Wagner, G.M., Chiu, D.T.Y., Yee, M.C., Lubin, B.H., 1986. Red cell vesiculation - a common membrane physiological event. Journal of Laboratory and Clinical Medicine 108, 315-324. 